\title{
La Ciencia política en URUguay: UN DESARROLLO TARDÍO, INTENSO Y ASIMÉTRICO
}

\author{
Adolfo Garcé* \\ Universidad de LA RePÚBLICA, URUGUAY
}

\begin{abstract}
Resumen
Las ciencias sociales en Uruguay han tenido un desarrollo tardío en comparación con las de otros países de la región. La ciencia política uruguaya se institucionalizó recién a fines de la década del 80 cuando, en el marco de la Universidad de la República, se formó un instituto de investigación especializado en esta temática y se comenzaron a dictar los primeros cursos de grado. Durante la década del 90 la ciencia política experimentó un despegue intenso tanto en el número de estudiantes matriculados como en actividades de investigación. Asimismo, rápidamente, los politólogos han conquistado un espacio importante en el análisis político y el debate público. Sin embargo, en este momento, la politología en Uruguay está enfrentada al desafío de corregir algunas asimetrías importantes.
\end{abstract}

\begin{abstract}
In Uruguay, social sciences have developed later than in other countries in the region. Political science, as an independent discipline, was only institutionalized at the end of the 1980 s when, within the University of the Republic, a specialized institute of research was established and began to offer undergraduate courses in the discipline. It is undeniable that during the 1990's political science experienced an intensive growth both in the number of students and research activities. At the same time, political scientists started to play an important role in the political analysis and public debate. Nevertheless, currently, Uruguayan political science faces the challenges of correcting some important asymmetries.
\end{abstract}

PALABRAS CLAVE - Ciencia Política - Uruguay - Disciplina - Instituciones • Opinión Pública • Desarrollo Asimétrico

\section{UNA DISCIPLINA QUE SE HIZO ESPERAR}

A pesar de que Uruguay ha experimentado un relativamente temprano proceso de modernización social y ha generado, también precozmente, un sistema universitario sofisticado y complejo, sus ciencias sociales han tenido un desarrollo comparativamente tardío (De Sierra, 2005). Si se toma como indicadores de consolidación de disciplinas científicas la formación de centros de investigación específicos y la consolidación de cursos de grado estables, se puede considerar que la

* Agradezco los comentarios y aportes de David Altman, Daniel Buquet, Jorge Lanzaro, Francisco Panizza, José Rilla y de los lectores anónimos de la Revista de Ciencia Política. 
economía comenzó en Uruguay en el primer lustro de la década del $50^{1}$, la sociología sobre finales de la década siguiente y la ciencia política, a finales de la del $80^{2}$.

Los esfuerzos académicos dirigidos a explicar el rezago de las ciencias sociales uruguayas respecto a las de otros países de la región como Argentina, Chile y Brasil han subrayado el peso de la visión conformista que prevaleció en Uruguay hasta que la crisis económica se hizo sentir. Según este argumento, la percepción de Uruguay como un país adelantado socialmente y aventajado culturalmente, muy extendida hasta mediados de la década del 50 , habría conspirado contra el desarrollo de las aproximaciones científicas a la realidad social (De Sierra, 2005).

La primera cátedra de ciencia política fue la de la Facultad de Derecho. Empezó a funcionar luego de la reforma del Plan de Estudios de 1957 bajo la dirección del Dr. Alberto Ramón Real3. Un poco más tarde, en el marco de la reforma del Plan de Estudios de 1966, fue creada la Cátedra de Ciencia Política de la Facultad de Ciencias Económicas, correspondiéndole a Carlos Real de Azúa la responsabilidad de iniciar la cátedra. Sin embargo, la ciencia política recién se institucionalizó en Uruguay después de la restauración de la democracia. La piedra fundamental en la construcción de esta disciplina fue la creación del Instituto de Ciencia Política de la Facultad de Derecho de la Universidad de la República, proceso que se concretó entre 1985 y 1989.

Para explicar por qué el despegue de la ciencia política uruguaya se registró tantos años después que el de la economía o la sociología vernáculas hay que buscar un poco más. Romeo Pérez Antón, en uno de los pocos textos que se plantean este pregunta, relaciona la ausencia de ciencia política con la muy alta calidad de la reflexión generada por los partidos políticos: "la ciencia política habría estado sustituida, según esta hipótesis, por ese óptimo de la discusión (práctica y parcial, pero lúcida)" (Pérez Antón, 1992: 42).

1 La creación del Instituto de Economía Bancaria y Monetaria a principios de los 50 (que se convertiría en 1963 en el actual Instituto de Economía de la Facultad de Ciencias Económicas) y la reforma del Plan de Estudios de 1954 (que encaminó la separación de los economistas respecto a los contadores) son momentos claves en el despegue de la economía en Uruguay. Los principales factores explicativos de este proceso hay que buscarlos en el impacto de la crisis económica que se hizo sentir durante el primer lustro de los 50 y en la penetración y difusión del pensamiento cepalino, que se difundió en Uruguay principalmente gracias a la influencia de Luis Faroppa. Durante la década del 60, el esfuerzo de planificación económica realizado en el marco de la "Alianza para el Progreso" legitimó muy fuertemente a la economía (Barbato, 1986; Garcé, 2005).

2 El auge de la sociología es posterior y menos intenso que el de la economía. La primera Cátedra de Sociología se creó en 1915, en la Facultad de Derecho de la Universidad de la República. Sin embargo, habrá que esperar hasta la década del 50 para que cobre un dinamismo importante. Entre 1956 y 1958, a iniciativa de Isaac Ganón (abogado), fue creado el Instituto de Ciencias Sociales en la Facultad de Derecho de la Universidad de la República. Durante los años 60, bajo el impulso de Aldo Solari (otro abogado), este Instituto se consolidó y estrechó relaciones con la academia internacional. Como testimonio de este esfuerzo cabe citar, por ejemplo, el Seminario organizado por Aldo Solari y Seymur Martín Lipset en 1964, en Montevideo, sobre "Elites en América Latina". A mediados de la década del 60 se incorporan al Instituto de Ciencias Sociales algunos sociólogos graduados en el exterior como Gerónimo de Sierra, Carlos Filgueira, Susana Prates y Néstor Campiglia. Simultáneamente, en torno a la figura del arquitecto J uan Pablo Terra (en el CLAEH y en la cátedra de sociología de la Facultad de Arquitectura), se consolidó otro polo de investigación en sociología. La licenciatura en sociología fue creada en 1968, y funcionó en la órbita de la Facultad de Derecho hasta 1989, cuando se integró a la Facultad de Ciencias Sociales (Filgueira, 1986; De Sierra, 2005).

3 Alberto Ramón Real, un abogado de prestigio, fue también Catedrático de Derecho Administrativo y Decano de la Facultad de Derecho. Según el testimonio de Jorge Lanzaro, siguiendo la línea predominante en la politología francesa en aquella época, Ramón Real enseñaba una ciencia política estrechamente vinculada al derecho público. Alfredo M. Errandonea, también abogado, ocupaba el cargo de Profesor Adjunto en esa cátedra. Introdujo el estudio de autores norteamericanos, especialmente los de la corriente estructural-funcionalista. 
J unto a esta hipótesis -sobre cuya validez no pretendo pronunciarme ahora- habría que considerar al menos dos más. En primer lugar, cabe preguntarse si la notable centralidad de los partidos en la vida del país no habrá tenido un efecto paradójico: es posible que en lugar de estimular el estudio científico de la política, lo haya inhibido. La lógica que subyace a esta hipótesis es la siguiente: la legitimación y el desarrollo de un conocimiento sereno y a-partidario sobre la política podrían inhibirse en contextos sociales extraordinariamente politizados, esto eso, en sociedades muy divididas por pertenencias políticas. Si se va por la pista correcta, el avance de la disciplina se habría visto dificultado por el auge de la militancia política y de la polarización ideológica de los 60 y facilitado por el contexto de "desencantamiento" y retracción de la política que siguió al entusiasmo cívico generado por la recuperación de la democracia en muchos países de la región.

En segundo lugar, el rezago de la ciencia política bien podría explicarse por el sesgo antipolítico que caracterizó a los principales abordajes en boga en las ciencias sociales de la década del 60 . La mayoría de los enfoques que prevalecían confiaban poco en la autonomía de la política. En realidad, para paradigmas tan variados como el marxismo -el paradigma más influyente en la intelectualidad uruguaya de los 60-, el estructuralismo cepalino -enfoque predominante entre los economistas- o la teoría de la modernización, para entender la política había que sumergirla en los procesos económicos y sociales.

Mirado desde este punto de vista, el nacimiento de la ciencia política estaría estrechamente vinculado con la crisis de los reduccionismos economicistas y de los enfoques socio-céntricos prevalecientes antes de la dictadura, y con la vigorosa recuperación de la noción de la autonomía de la política que se verifica al interior de la pequeña pero entusiasta comunidad de cientistas sociales compatriotas durante el autoritarismo.

\section{LOS PRIMEROS PASOS: AUTORES, TEMAS, ENFOQUES}

La ciencia política uruguaya de los 90 no nació ex nihilo. En realidad, se apoyó en una fuerte acumulación previa que se remonta al siglo XIX. Entre los antecedentes más relevantes, junto a las más refinadas expresiones del pensamiento de los propios partidos políticos que ha subrayado Pérez Antón, hay que reservar un lugar de privilegio para los principales cultores de la historia. Entre ellos, durante la década del 40, se destaca el aporte de J uan Pivel Devoto y, durante los 70 y 80 , algunas de las obras fundamentales de J osé Pedro Barrán y Benjamín Nahum, entre otros (Caetano y Rilla, 1992).

Durante los años 60 hubo algunos esfuerzos solitarios, pero relevantes por construir una perspectiva politológica sobre el caso uruguayo. Carlos Real de Azúa, abogado, profesor de literatura y estética, crítico literario e historiador, autodidacta empedernido y ensayista brillante, es considerado el principal iniciador de la ciencia política en el país. Junto a él, desde la perspectiva de la sociología política, Aldo Solari, otro abogado, realizó aportes muy relevantes para la comprensión de algunas lógicas del sistema político uruguayo (Pérez Antón, 1986).

La producción de Real de Azúa fue y sigue siendo una referencia obligatoria para los estudiosos de la política uruguaya. Aunque escribió mucho y sobre temas variados, la cuestión del poder político (quiénes lo ejercen y cómo) lo fascinó muy especialmente. En su aproximación a este tópico, en términos teóricos, estuvo mucho más cerca de Max Weber y de los teóricos del elitismo (Mosca, 
Pareto, Michels) que de Robert Dahl y los pluralistas. En el plano metodológico, fue evolucionando desde los ensayos de corte histórico sobre el caso uruguayo hasta la política comparada.

Aldo Solari, uno los padres de la sociología, es también una figura crucial en el estudio científico de la política en Uruguay. Igual que Real de Azúa, Solari tenía un profundo conocimiento de la trayectoria histórica del sistema político uruguayo y de sus partidos tradicionales. Sin embargo, ambos enfoques privilegiaban dimensiones analíticas distintas. Mientras que, en sintonía con las teorías elitistas, Real de Azúa prestaba especial atención a la "clase dirigente", Solari ponía su foco en las pautas de cultura política prevalecientes en la sociedad y en sus consecuencias en el terreno político en general y, muy especialmente, en el plano del comportamiento electoral.

Uno de sus temas favoritos fue, pues, el estudio del clientelismo. Solari hacía remontar sus causas hasta la cultura política particularista predominante en el país y visualizaba sus consecuencias negativas tanto en las características de la burocracia pública (escaso profesionalismo, baja productividad) como en las de los partidos políticos (cortoplacisimo, debilidad en la elaboración programática). En el fondo, la visión de Aldo Solari era profundamente pesimista. Los partidos uruguayos no estaban en condiciones de llevar adelante el programa de la modernización desarrollista, porque la sociedad uruguaya prefería las soluciones particulares a los programas universalistas y los criterios científicos.

En esta visión sombría de la política uruguaya, ciertamente, no estaba solo. Muy por el contrario, lo que mejor caracteriza a la producción de los intelectuales uruguayos de las dos décadas previas al golpe de 1973 es su notorio desencanto respecto a la política doméstica. Para Solari y para Real de Azúa, para Quijano y sus colaboradores en la revista Marcha, para los intelectuales terceristas ${ }^{4}$, pero también para los comunistas, la política uruguaya en general y los viejos partidos tradicionales en particular, atravesaban una crisis muy profunda y, como todo en aquella época, eminentemente "estructural".

\section{INSTITUCIONES Y ACTORES EN LA FORJA DE LA CIENCIA POLÍTICA URUGUAYA}

La dictadura interrumpió bruscamente el proceso de acumulación en ciencias sociales que se venía realizando al interior de la Universidad de la República (en el Instituto de Economía de la Facultad de Ciencias Económicas y en el Instituto de Ciencias Sociales de la Facultad de Derecho). La intervención de la universidad y la expulsión de los docentes relacionados con organizaciones de izquierda de la enseñanza oficial obligó a la gran mayoría de los economistas y sociólogos a construir centros privados de investigación. En este contexto, en 1974 se revitalizó el Centro Latinoamericano de Economía Humana (CLAEH). En 1975 fueron fundados el Centro de Información y Estudios Sociales del Uruguay (CIESU) y el Centro de Investigaciones Económicas (CINVE). En 1977 apareció también el Centro Interdisciplinario de Estudios sobre el Desarrollo del Uruguay (CIEDUR). Estas instituciones desempeñaron un papel clave en el desarrollo de las ciencias sociales durante la dictadura (De Sierra, 2005).

4 Durante la segunda posguerra buena parte de la intelectualidad uruguaya se autodefinía como "tercerista": tanto EEUU como la URSS eran considerados portadores de designios imperialistas. 
El aporte de algunos de estos centros privados al fortalecimiento de la ciencia política fue muy importante. CIESU acogió a Carlos Real de Azúa, y apoyó la primera fase de las carreras profesionales de Luis Eduardo González, Carina Perelli y J uan Rial. EI CLAEH organizó, a comienzos de los 80, el primer grado en ciencia política del país. También desarrolló un programa de investigación muy fecundo sobre los partidos políticos y la construcción del pluralismo uruguayo, con la participación de especialistas en distintas disciplinas como Gerardo Caetano, Pablo Mieres, Horacio Martorelli, Carlos Pareja, Romeo Pérez Antón, J osé Rilla y Carlos Zubillaga, entre otros (Pérez Antón, 1992: 49-52). En CIEDUR, especialmente entre 1980 y 1984, César Aguiar y otros colegas investigaron sobre partidos políticos, Estado y sociedad, con énfasis en las lógicas de representación de intereses de grupos sociales y clientelas políticas. (Pérez Antón, 1999). La creación, en 1986, de la Sociedad de Análisis Político animada, entre otros, por César Aguiar (CIEDUR), Alfredo Errandonea ( $h$ ), Carlos Filgueira (CIESU) y Gerónimo de Sierra (CIEDUR) constituye un indicador del avance de los estudios enfocados en el sistema político uruguayo durante estos años (De Sierra, 2005) .

Al mismo tiempo, en el exterior del país, se iba formando la primera generación de doctores en ciencia política: Francisco Panizza hizo su PhD en Essex, Luis Eduardo González en Yale, Fernando López-Alves en California, Jorge Lanzaro y Luis Costa Bonino en Paris ${ }^{6}$.

Apoyándose en la acumulación realizada en los mencionados centros privados y en el aporte de algunos académicos formados en el exterior (en programas de Doctorado o de Maestría) se conformó, en un proceso que comenzó a fines de 1985, el Instituto de Ciencia Política (ICP). Al principio funcionó simplemente como mecanismo de coordinación de los profesores de la Cátedra de Ciencia Política de la Facultad de Derecho ${ }^{7}$. Al formarse la Facultad de Ciencias Sociales en 1989, el ICP se incorporó a ella como uno de sus departamentos académicos. Pronto cobró un impulso importante: en 1989 se dictó el primer curso de grado de ciencia política de la Universidad de la República, en 1997 la primera Maestría en Ciencia Política y, finalmente, en el 2005, empezó a funcionar el primer programa de Doctorado en Ciencias Sociales de la Facultad de Ciencias Sociales (Universidad de la República) ${ }^{8}$.

Durante todo este período, pero muy especialmente entre 1985 y 2000, la evolución del ICP ha estado estrechamente unida al liderazgo de Jorge Lanzaro, que jugó un papel protagónico en la fundación de esta institución y en el proceso posterior de organización de su actividad (diagramando los primeros programas de investigación, generando las primeras publicaciones, entre ellas, la

Un buen panorama del estado de desarrollo de la ciencia política en Uruguay al restablecerse la democracia leyendo los artículos escritos por uruguayos en el número que la Revista Mexicana de Sociología dedicara especialmente a examinar la transición uruguaya (XLVII, 1985).

6 Panizza trabajó unos años en el ICP y luego volvió a emigrar. En este momento enseñan política latinoamericana en The London School of Economics and Political Science. Costa Bonino también colaboró durante algunos años con el ICP dictando un curso de política comparada. Ahora se ha especializado en marketing político y tiene una consultora. Fernando López-Alves se radicó tempranamente en EEUU y Buenos Aires. Ahora enseña política comparada en la Universidad de Santa Bárbara en California. Lanzaro y Luis Eduardo González luego de terminar sus doctorados se radicaron en Uruguay. El primero, como se verá un poco más adelante, se concentró en la construcción del ICP. EI segundo se dedicó al trabajo profesional en el campo de la opinión pública.

7 A partir de la restauración de la democracia, concurso mediante, Julio Barreiro, J uan Rial y J orge Lanzaro ocuparon la cátedra de ciencia política de esta Facultad.

8 Este doctorado admite diversas especializaciones, entre ellas, ciencia política. 
Revista Uruguaya de Ciencia Política ${ }^{9}$, organizando los primeros seminarios, gestionando las primeras consultorías). Un acierto muy importante en su gestión fue el de haber amalgamado distintas corrientes teóricas, ideológicas y políticas, estimulando la conformación de un ambiente pluralista, altamente beneficioso para el debate y el aprendizaje académico.

Al calor de esas discusiones, se terminó de gestar una profunda revisión de la visión "crítica" ("sombría", se dijo más arriba) de la política uruguaya que había prevalecido durante los años 50 y 60. Dos ideas, ambas muy arraigadas en la visión "crítica", fueron muy especialmente impugnadas. En primer lugar, se desmontó la visión negativa del sistema electoral uruguayo y en su lugar, se propuso una relectura radical de la historia de las instituciones políticas y de los partidos uruguayos. En segundo lugar, fue duramente cuestionada la idea de la "improductividad" del sistema político uruguayo: la reflexión sobre el "bloqueo político" fue sustituida por un estudio (y un elogio) de la lógica gradualista que la conformación pluralista del sistema político uruguayo le impone a su policy-making process ${ }^{10}$.

Desde marzo del 2000 hasta mayo del 2005 ejerció la dirección del ICP Gerardo Caetano. Profesor de historia y analista político, Caetano participó muy activamente en los programas de historia y ciencia Política del CLAEH durante los 80 y en el período de forja y consolidación del ICP. Gracias a su impulso, durante los últimos cinco años el ICP ha incrementado notoriamente su comunicación con la sociedad uruguaya. El aumento de la frecuencia de las publicaciones y el fuerte incremento del número de convenios y consultorías constituyen indicadores de esta expansión ${ }^{11}$.

\section{LAS CONSULTORAS DE OPINIÓN PÚBLICA Y LA LEGTIMACIÓN DE LA CIENCIA POLÍTICA}

Ciertamente, no sólo el ICP aportó a la forja de la ciencia política. Otras instituciones (por ende, otros académicos) también contribuyeron a esta tarea. En especial, el trabajo de las principales consultoras de opinión pública, jugó un papel relevante en la legitimación del abordaje científico de los asuntos políticos.

En realidad, la investigación de la opinión pública en Uruguay había comenzado muchas décadas atrás a instancias del Instituto Uruguayo de Opinión Pública (IUDOP). Entre la fundación del IUDOP (1956) y el quiebre de la democracia (1973) se realizaron diversos estudios de opinión pública en empresas privadas (IUDOP, GALLUP) y centros académicos (Instituto de Ciencias Sociales de la Facultad de Derecho). Sin embargo, no llegaron a tener ni la sistematicidad ni la visibilidad que cobrarían a partir de la restauración de la democracia. Las elecciones de 1984, precisamente, marcaron un hito en este campo de estudios (Aguiar, 2000).

9

La Revista Uruguaya de Ciencia Política se empezó a publicar en 1991. Hasta la fecha se editaron 14 números. Sus directores son Gerardo Caetano y J orge Lanzaro, y su editor es Javier Gallardo. Los directores y el editor toman las decisiones acerca de qué artículos se publican. No existen hasta la fecha otros mecanismos de arbitraje.

10 Los trabajos publicados en Lanzaro (2000) constituyen buenos ejemplos del enfoque "revisionista" predominante en el ICP durante estos años. Otras obras publicadas en la época apuntan en direcciones similares. Entre ellas se destaca Fragmentación política y gobierno en Uruguay. Un enfermo imaginario, de Daniel Buquet Daniel, Daniel Chasquetti y J uan Andrés Moraes, Facultad de Ciencias Sociales, 1998. Ver la reseña de Jones, Mark (1999).

11 A partir de mayo de 2005, la dirección del ICP pasó a ser ocupada por la Dra. Constanza Moreira. 
Durante las dos últimas décadas, las encuestas de opinión pública y sus responsables han cobrado un protagonismo importante, especialmente durante el año electoral ${ }^{12}$. En general, más allá de cuestionamientos y debates, las encuestas de opinión pública son consideradas herramientas confiables tanto por el público como por los políticos. De todos modos, el peso que han adquirido la información y los datos proporcionados por los principales expertos en opinión pública genera reacciones y cuestionamientos entre los actores políticos, especialmente cuando éstos interpretan que los resultados divulgados afectan sus intereses y posibilidades electorales (Buquet, 2004).

El prestigio que en el desempeño de esta tarea han logrado conquistar los responsables de las principales firmas de opinión pública ha operado beneficiosamente sobre la ciencia política: independientemente que su responsable sea un profesional titulado en esta disciplina, cada vez que se comprueba (la sociedad comprueba) que una consultora de opinión pública acertó en una medición 0 un pronóstico, se genera un plus de credibilidad y legitimidad que recae en la ciencia política. La explicación de este fenómeno es muy sencilla: habitualmente estos expertos son presentados al público como "politólogos" y no como sociólogos o especialistas en opinión pública ${ }^{13}$.

\section{EL INSTITUTO DE CIENCIA POLÍTICA HOY}

El ICP es el principal centro de referencia en ciencia política del país. Cuenta con un plantel numeroso, estable y calificado de profesionales que investigan, enseñan grado y postgrado y participan habitualmente en el debate público. En la planilla del ICP aparecen 65 nombres. La tercera parte, aproximadamente, realizan sólo tareas docentes. Del medio centenar restante, una decena están en régimen de Dedicación Total (full-time).

Durante los últimos diez años ha aumentado fuertemente el grado de formación de los docentes e investigadores del ICP. Numerosos colegas obtuvieron la maestría en ciencia política que ofrece la propia FCS. Muchos otros optaron por programas de Doctorado en el exterior. En esta segunda generación de doctores en ciencia política prevaleció nítidamente la preferencia por universidades latinoamericanas: la mayoría (casi una decena) se doctoraron en IUPERJ (Brasil), pero otros colegas optaron o por FLACSO (México) o por la UBA (Argentina). Muy pocos, en cambio, optaron por doctorados en universidades de EEUU o Europa ${ }^{14}$. Finalmente, dos colegas realizaron el Doctorado en Ciencias Humanas -con énfasis en ciencia política- que ofreció la Universidad Católica de Montevideo ${ }^{15}$.

Un indicador claro de la consolidación de la ciencia política en Uruguay es el incremento de las matrículas en esta disciplina. El número de ingresos a la carrera de ciencia política de la Universidad de la República ha ido creciendo hasta, en algunos años, alcanzar el nivel de sociología.

12 Las cinco principales firmas son: Equipos/Mori (hace encuestas políticas desde 1984), Factum (1989), Cifra (1992), Interconsult (1993) y Radar (1998). No se puede explicar el rápido auge de la ciencia política en Uruguay sin el prestigio social conquistado por los responsables de estas firmas. Entre ellos cabe mencionar a César Aguiar y Agustín Canzani (Equipos/Mori), Luis Eduardo González (Cifra), Oscar Bottinelli (Factum) y J uan Carlos Doyenart (Interconsult).

13 Un indicador indirecto de esto es que se ha constatado la existencia de un "ciclo político" en el ingreso a la licenciatura de ciencia política. El número de ingresos crece más en el año posterior a la elección. Puede revisarse esta hipótesis con la información de la tabla "Evolución de los ingresos a la Facultad de Ciencias Sociales". Téngase en cuenta que las elecciones nacionales se realizaron en 1994 y 1999. Nótese el fuerte incremento de los ingresos en 1995 respecto a 1994 y en el 2000 respecto a 1999.

14 Entre los más jóvenes viene creciendo la expectativa de postularse a programas de doctorado en EEUU.

15 Hasta la fecha se han recibido cuatro personas en el Doctorado de la Universidad Católica del Uruguay. Es un doctorado en ciencias humanas que admite distintos énfasis (sociología, educación, ciencia política, entre otros). En este momento el programa de doctorado está cerrado. 
TABLA 1: Evolución del número de ingresos a la Facultad de Ciencias Sociales

\begin{tabular}{lrrrrrrrrrrrrrrr}
\hline Licenciatura & 1992 & 1993 & 1994 & 1995 & 1996 & 1997 & 1998 & 1999 & 2000 & 2001 & 2002 & 2003 & 2004 \\
\hline Ciencia Política & 115 & 67 & 72 & 147 & 106 & 121 & 119 & 150 & 243 & 230 & 206 & 253 & 174 \\
Sociología & 268 & 152 & 241 & 193 & 205 & 230 & 205 & 183 & 247 & 250 & 222 & 309 & 235 \\
Trabajo social & 231 & 139 & 162 & 168 & 166 & 187 & 231 & 250 & 312 & 337 & 397 & 482 & 472 \\
Total & 614 & 358 & 475 & 508 & 477 & 538 & 555 & 583 & 802 & 817 & 825 & 1044 & 881 \\
\hline
\end{tabular}

Fuente: Anuario 2004 (FCS).

TABLA 2: Evolución del número de egresos de la Facultad de Ciencias Sociales

\begin{tabular}{lrrrrrrrrr}
\hline Licenciatura & 1995 & 1996 & 1997 & 1998 & 1999 & 2000 & 2001 & 2002 & 2003 \\
\hline Ciencia Política & 4 & 4 & 5 & 6 & 6 & 9 & 13 & 10 & 11 \\
Sociología & 67 & 57 & 46 & 32 & 26 & 20 & 20 & 26 & 27 \\
Trabajo social & 84 & 97 & 35 & 16 & 19 & 14 & 23 & 18 & 24 \\
Total & 155 & 158 & 86 & 54 & 51 & 43 & 56 & 54 & 62 \\
\hline
\end{tabular}

Fuente: Anuario 2004 (FCS)

Empero, el número de graduados por año apenas se acerca a la decena. Es evidente que la proporción de egresos en el total de ingresos es muy baja. En total, hasta el año 2004, egresaron de la licenciatura 68 politólogos. A estos hay que sumarles otros 21 que se han graduado en la Facultad de Ciencias Humanas de la Universidad Católica ${ }^{16}$.

A la consolidación y expansión del grado, hay que agregar la puesta en marcha de algunos postgrados. Hasta la fecha se han realizado tres promociones de la maestría en ciencia política (1997-1999, 2000-2001 y 2002-2003) y se encuentra en curso la cuarta promoción (20042005). Hasta la fecha, son 16 los candidatos que defendieron sus tesis y obtuvieron el título de Magíster en Ciencia Política. A comienzos de 2005 las ciencias sociales uruguayas han dado otro paso importante en su proceso (tardío pero enérgico) de expansión: se puso en marcha el programa de doctorado de la Facultad de Ciencias Sociales de la Udelar.

También, paulatinamente, se ha incrementado el abanico de temas investigados. Entre otros, se destacan los polos de acumulación siguientes: i) Partidos, régimen de gobierno y sistemas electorales (con una fuerte acumulación empírica acerca de procesos de gobierno en el presidencialismo y efectos de sistemas electorales); ii) Estructuras, capacidades y reforma del Estado (predomina una lectura crítica del New Public Managment); iii) Instituciones, ideas e intereses en el cambio en las políticas sociales (existe una variedad de enfoques, desde los que se instalan en las teorías de redes a los que registran dinámicas de paradigmas de políticas y procesos de policy-transfer); iv) Teoría Política (con puntos altos en el estudio del republicanismo en Uruguay y teorías feminis-

16 En 1991 empezó a funcionar la licenciatura en ciencia política en la Universidad Católica. Los primeros egresos se registraron en 1998. Cinco de ellos han continuado sus estudios en el exterior (EEUU, Canadá y Bélgica). Esta información fue aportada por el Programa de Información Estratégica de UCUDAL. 
tas) ${ }^{17}$; v) Historia Política (con especial énfasis en la historia de los partidos y en la reconstrucción de la "memoria" del proceso autoritario) ${ }^{18}$.

En un marco general tan amplio, llama la atención, cuando se toma un poco de distancia, tres ausencias. En primer lugar, a diferencia de lo que ocurre en otros medios académicos, no existe en el ICP una acumulación en estudios de política exterior. Cabe señalar, de todos modos, durante los últimos años se organizó un grupo de estudios sobre MERCOSUR. En segundo lugar, tampoco se registra una acumulación significativa en el estudio de las políticas públicas no sociales. Casi no existen estudios politológicos (ya sean Tesis, investigaciones corrientes o consultorías) acerca de políticas agropecuarias, industriales 0 de ciencia y tecnología ${ }^{19}$. En tercer lugar, en el ICP tampoco se ha podido consolidar hasta la fecha un núcleo potente de investigaciones en el campo de la economía política, pese a algunos valiosos intentos en ese sentido ${ }^{20}$.

No sólo existe una variedad importante en los objetos de estudio. En el ICP conviven (no siempre pacíficamente) diferencias en el plano de los métodos y en el de los paradigmas. La preocupación por construir una ciencia política empírica, "positiva", alejada del ensayismo, se ha intensificando, a medida que se completaba el proceso de formación de lo que más arriba denominamos la segunda generación de doctores. Por otro lado, en el terreno del debate teórico, también en Uruguay se ha verificado el ascenso del institucionalismo rational choic $e^{21}$. De todos modos este enfoque está lejos de ser hegemónico.

A diferencia de lo que ocurrió con la primera generación de doctores en ciencia política que crearon y cultivaron sólidos vínculos especialmente con especialistas y centros de investigación europeos, la nueva generación de politólogos está claramente más preocupada por leer las revistas publicadas en EEUU que por tomar contacto con la producción de Europa. Esta preferencia hacia el establecimiento de vínculos con los especialistas residentes en EEUU se manifiesta, por ejemplo, en la activa participación de los politólogos uruguayos en los congresos de LASA.

\section{LOS DESAFÍOS DE UN DESARROLLO ASIMÉTRICO}

El desarrollo de la ciencia política en Uruguay, a partir de 1989 hasta ahora, ha sido intenso y fructífero. En 15 años se consolidó la enseñanza de grado y de postgrado, se constituyeron

17 Pablo da Silveira, el más prolífico entre los estudiosos uruguayos en el campo de la teoría política, no trabaja en el ICP de la Universidad de la República sino en la Universidad Católica.

18 Esta descripción se apoya en el Informe de actividades de investigación 2000-2004, elaborado por la Coordinación de Investigación del ICP, firmado por Adolfo Garcé y Carlos Luján (abril 2005).

19 La reciente tesis de maestría sobre las políticas de ciencia y tecnología en Uruguay, de nuestro colega Martín Rivero en el Institut of Social Studies (ISS) de La Haya, es la única excepción.

20 Entre las aproximaciones más enérgicas a esta temática se destacan algunos trabajos de Juan Andrés Moraes y de Fernando Filgueira. Ver: Aboal y Moraes (2003).

21 Sobre las corrientes neoinstitucionalistas ver: Hall, Peter y Rosemay Taylor. 
importantes programas de investigación, se tejieron redes académicas de calidad y se realizaron publicaciones que innovaron en la comprensión de los fenómenos políticos en Uruguay 22.

De todos modos, el crecimiento de la ciencia política uruguaya muestra algunas asimetrías preocupantes. En primer lugar, los politólogos uruguayos son mucho más escuchados y leídos en Uruguay que entre sus pares de América Latina y del resto del mundo. La mayor parte de las publicaciones realizadas se ocupan del caso uruguayo (los estudios comparativos son poco frecuentes), se realizan en Montevideo y circulan fundamentalmente dentro de fronteras. De todos modos, se viene intensificando la práctica de publicar en revistas de la región (generalmente de Argentina, México o Chile). Sin embargo, hasta la fecha, no existen publicaciones de académicos del ICP en revistas del Social Sciences Citation Index ${ }^{23}$.

TABLA 3: Entradas en el SSCI de politólogos uruguayos (1975-2005)

\begin{tabular}{llll}
\hline \multicolumn{2}{c}{ Residentes en Uruguay } & Investigan en otro país & Total \\
\hline Investigan en ICP & No investigan en ICP & \\
\hline
\end{tabular}

0 5

Como corolario natural de la preferencia por las publicaciones domésticas 0 , en el mejor de los casos, regionales, los trabajos de los politólogos del ICP son poco citados por los colegas de otras regiones.

TABLA 4: Citas recibidas en Journals ISI de politólogos uruguayos (1975-2005)

\begin{tabular}{llll}
\hline \multicolumn{2}{c}{ Residentes en Uruguay } & Investigan en otro país & Total \\
\hline Investigan en ICP & No investigan en ICP & \\
\hline
\end{tabular}
65 91 160 316

En segundo lugar, los politólogos uruguayos son mucho más demandados por los medios de comunicación (generalmente para comentar y explicar procesos electorales) que por instituciones (ya sean agencias del Estado u ONG) involucradas en la elaboración y gestión de políticas públicas. Todavía hoy, el politólogo es visto más como un experto en elecciones y partidos políticos

22 Para tener una idea de la evolución teórica y metodológica (y de la renovación generacional) de la ciencia política uruguaya desde la fundación del ICP hasta el 2004 conviene comparar dos publicaciones emblemáticas. El punto de partida queda bien reflejado en la obra Los partidos políticos de cara al 90 (FCU-ICP-FESUR), publicada en diciembre de 1989, que reúne las versiones corregidas de un Seminario realizado en junio de 1989 en el que participaron los principales animadores de la ciencia política uruguaya de la época. El estado actual queda de manifiesto en la obra La izquierda uruguaya: entre la oposición y el gobierno, coordinada por Jorge Lanzaro y publicada en Montevideo noviembre de 2004 por Editorial Fin de Siglo.

23 Se realizó, especialmente para este artículo, un relevamiento de las publicaciones de politólogos uruguayos en ISI-Web of Knowledge, utilizando la base de datos "Social Science Citation Index" (SSCI). De acuerdo a esta información, entre 1975 y 2005, los politólogos uruguayos que publican en revistas indexadas son los que no trabajan en el ICP de la Universidad de la República. Entre los que residen en el exterior aparecen en el SSCI J uan Rial, Carina Perelli, Francisco Panizza, Hugo Borsani, Alberto Spektorowski, David Altman, Rossana Castiglioni, Fernando López-Alves y J uan Pablo Luna. Entre los académicos que viven en Uruguay pero no investigan en el ICP se registran algunos artículos de Luis $E$. González, César Aguiar y Carlos Filgueira. 
que como un analista de políticas públicas. No es un problema menor, en un país que precisa rápidamente profesionalizar la gestión pública y mejorar la interfaz entre conocimiento especializado y políticas públicas (De Armas y Garcé, 2000). La expansión del número de funcionarios del ICP es una prueba cabal de los problemas que existen para activar una demanda enérgica de politólogos desde la sociedad y el Estado.

En tercer lugar, existe una asimetría importante entre el prestigio social y la visibilidad pública que ha logrado acumular la ciencia política y los bajos niveles de ingreso promediales de sus cultores. Un profesor titular ${ }^{24}$ (Grado 5) full time gana aproximadamente US $\$ 1.400$ por mes (antes de impuestos) y US $\$ 750$ por una carga horaria de 40 horas semanales. Téngase en cuenta que el proceso de ingreso al régimen de full time es exigente y lento. Además, aunque han ido creciendo, los fondos concursables destinados a financiar investigaciones científicas son todavía muy escasos.

Last but not least, impacta y preocupa el contraste entre la relativa facilidad con la que, pese a la inexistencia de un sistema de becas que facilite la salida a programas de postgrados en el exterior, los graduados en ciencia política en Uruguay son admitidos en buenos programas de doctorado a nivel internacional y sus graves dificultades para retornar al país e insertarse dignamente en el mercado laboral ${ }^{25}$. Como vimos más arriba, de la primera generación de doctores en ciencia política solamente J orge Lanzaro pudo consagrarse plenamente a la vida académica nacional. La segunda generación tuvo otras posibilidades de inserción profesional, entre otras razones, porque cubrió las plazas docentes generadas por la creación (y ulterior expansión) de las actividades de enseñanza durante la fase de consolidación del ICP. Sin embargo, el proceso de reinserción en la vida académica uruguaya de quienes optan por ir a terminar su formación en centros de excelencia se ha vuelto cada vez más complicado. Paradójicamente, no existe hasta la fecha ninguna política pública enérgica dirigida a evitar la "fuga de cerebros", facilitando la rápida reinserción -en ámbitos académicos 0 agencias relevantes en los procesos de gobierno y policy-making- de los uruguayos posgraduados en el extranjero. Éste es, seguramente, uno de los desafíos mayores para una ciencia política nacional que, en su corta vida, ha dado muestras de pasión por su objeto de estudio, dinamismo y vocación por la excelencia.

\section{REFERENCIAS}

Aguiar, César. 2000. "La Historia y la historia: Opinión Pública y opinión pública en el Uruguay”. Prisma 15.

AAVV. 1989. Los partidos políticos de cara al 90. Montevideo: FCU-ICP-FESUR.

AAV. 2000. Elecciones 1999/2000. Montevideo: ICP-FCS. EBO.

AAVV. 2003. La Política (Tomo II). Montevideo: EBO, Serie "El Uruguay del Siglo XX".

AAVV. 2002. Dinero y política, El financiamiento de los partidos en la democracia. Montevideo: EBO.

Aboal, Diego y Juan Andrés Moraes (coord.). 2003. Economía y Política en Uruguay, Instituciones y actores políticos en el proceso económico. Montevideo: Ediciones Trilce.

Barbato, Celia. 1986. "Economía”. En Ciencia y Tecnología en Uruguay, editado por autores varios. Montevideo: Ministerio de Educación y Cultura - CINVE.

24 La carrera docente en la Universidad de la República tiene cinco escalones: Grado 1: Profesor Ayudante; Grado 2: Profesor Asistente; Grado 3: Profesor Adjunto; Grado 4: Profesor Agregado; Grado 5: Profesor Titular. El Grado 5 es, por tanto, el escalón más alto de la carrera docente.

25 No existen estudios sistemáticos de este punto. Sin embargo, existe cierto consenso entre los colegas del ICP en cuanto a que es más fácil lograr ser admitido en un buen programa de doctorado que regresar al país e insertarse adecuadamente. 
Borsani, Hugo. 2003. Eleições e Economia. Belo Horizonte: Editora UFMG/IUPERJ .

Buquet, Daniel, Daniel Chasquetti y J uan Andrés Moraes. 1998. ¿Un enfermo imaginario? Fragmentación política y gobierno en Uruguay, Montevideo: FCS- ICP.

Buquet, Daniel. 2004. "Public Opinion and Electoral Behavior in Uruguay". En Public Opinion and Polling around the World, editado por J ohn Geer. Santa Bárbara: ABC- CLIO.

Caetano, Gerardo y J osé Rilla. 1992. "Historia. Apuntes para una balance de treinta años de historia de partidos". En Partidos y electores: centralidad y cambios, editado por Gerardo Caetano, J osé Rilla, Pablo Mieres y Romeo Pérez. Montevideo: CLAEH-EBO.

Caetano, Gerardo (coord.). 1992. La alternativa parlamentarista. Montevideo: CLAEH-ECS.

Caetano, Gerardo, Javier Gallardo y José Rilla. 1996. La izquierda uruguaya: innovación, tradición y política. Montevideo: Ediciones Trilce.

Caetano, Gerardo, J osé Rilla, Pablo Mieres y Romeo Pérez. 1992. Partidos y electores: centralidad y cambios. Montevideo: CLAEH - EBO.

Costa Bonino, Luis. 1995. La Crisis del Sistema Político Uruguayo. Partidos Políticos y Democracia hasta 1973. Montevideo: FCU. De Armas y Garcé (coord.). 2000. Técnicos y Política. Montevideo: Ediciones Trilce.

De Sierra, Gerónimo. 2005. "Social Sciences in Uruguay (specifically in Sociology)". Social Science Information 44 (forthcoming).

Filgueira, Carlos. 1986. "Sociología". En Ciencia y Tecnología en Uruguay, editado por autores varios. Montevideo: Ministerio de Educación y Cultura - CINVE.

Garcé, Adolfo. 2005. "Economics, Economists and Politics in Uruguay". En Economists in the Americas, editado por John Markoff y Verónica Montecinos. Cheltenham and Northampton: Edward Elgar Publishing, forthcoming.

Garcé, Adolfo, 2002. Ideas y competencia política en Uruguay (1960 -1973). Revisando el "fracaso" de la CIDE. Montevideo: Ediciones Trilce.

Garcé, Adolfo y J aime Yaffé. 2004. La Era Progresista. Montevideo: Fin de Siglo.

Gillespie, Charles. 1995. Negociando la democracia. Políticos y generales en Uruguay. Montevideo: ICP-FCU.

Goiscia, Laura (coord.). 2001. Ciudadanía en Tránsito: perfiles para el debate. Montevideo: EBO.

González, Luis Eduardo et al. 1999. Los partidos políticos uruguayos en tiempos de cambio. Montevideo: UCUDAL - Fundación Banco de Boston.

González, Luis Eduardo. 1993. Estructuras políticas y democracia en Uruguay. Montevideo: FCU-ICP.

Lanzaro, Jorge (coord.). 2000. La "segunda" transición en el Uruguay. Montevideo: FCU-ICP.

Lanzaro, Jorge (comp.). 2001. Tipos de Presidencialismo y Coaliciones Políticas en América Latina. Buenos Aires: CLACSO - ASDI.

Lanzaro, Jorge (coord.). 1998. El fin del siglo del corporativismo. Caracas: Nueva Sociedad.

Lanzaro, Jorge (coord.). 2004. La izquierda uruguaya entre la oposición y el gobierno. Montevideo: Fin de Siglo.

Laurnaga, María Elena. 2001. La Geografía de un cambio. Montevideo: EBO.

Mancebo, María Ester y Conrado Ramos (coords). 2002. Uruguay: La reforma del Estado y las políticas públicas en la democracia restaurada (1995-2000). Montevideo: EBO.

Midaglia, Carmen. 2001. Alternativas de protección a la infancia carenciada. La peculiar convivencia de lo público y lo privado en Uruguay. Buenos Aires: Clacso.

Moreira, Constanza. 1997. Democracia y Desarrollo en Uruguay. Montevideo: ICP/CSIC/Ediciones Trilce.

Moreira, Constanza. 2004. Final de juego. Del bipartidismo tradicional al triunfo de la izquierda en Uruguay. Montevideo: Ediciones Trilce.

Moreira, Constanza y Susana Mallo. 2000. La larga espera: itinerarios de las izquierdas en Argentina, Brasil y Uruguay. Montevideo: Ediciones Trilce.

Panizza, Francisco. 1990. Uruguay, batllismo y después. Pacheco militares y tupamaros en la crisis del Uruguay batllista. Montevideo: EBO.

Pérez Antón, Romeo. 1986. "Ciencia Política". En Ciencia y Tecnología en Uruguay, editado por autores varios. Montevideo: Ministerio de Educación y Cultura - CINVE. 
Pérez Antón, Romeo. 1992. "Ciencia Política". En Partidos y electores: centralidad y cambios, editado por Gerardo Caetano, J osé Rilla, Pablo Mieres y Romeo Pérez. Montevideo: CLAEH - EBO.

Ramos, Conrado (coord.). 2003. La reconstrucción gerencial del Estado: enfoques políticos sobre la Nueva Gestión Pública. Montevideo: EBO.

Adolfo Garcé es MA en Ciencia Política. En la actualidad se desempeña como docente del Instituto de Ciencia Política, Facultad de Ciencias Sociales, Universidad de la República, Uruguay.

(E-mail: agarce@fcs.edu.uy). 